\title{
Generalization of Martinelli-Nelson method of pressure drop calculation in two-phase flows
}

\author{
Marian Trela*, Roman Kwidzinski, and Marcin Lackowski \\ The Szewalski Institute of Fluid-Flow Machinery, Polish Academy of Sciences, Fiszera 14, 80-231 Gdansk, Poland
}

\begin{abstract}
A simple method of pressure drop calculation for two-phase flows of different fluids during convective boiling in channels is presented. It is based on experimental data of pressure drop multiplier $R$ and void fraction $\varphi$ obtained by Martinelli and Nelson for boiling of water in vertical tubes. The data cover the whole two-phase domain from ambient to critical pressure. Unfortunately, they have been presented in graphical forms. The first step in the procedure proposed in the paper was a transformation of the graphical data into analytical formulas which contain such dimensionless quantities as steam quality $x$, Martinelli parameter $X$, multiplier $\Phi t^{2}$ and dimensionless coefficients $D, m, E$ and $k$. In the second step, simple analytical formulas were determined to express the dimensionless coefficients as a function of physical property parameter $K$. In this way two simple analytical expressions for the multiplier $R$ and void fraction $\varphi$ were obtained. They are in analytical dimensionless form so they may be used directly for different fluids, not only for water. This is the main advantage of the proposed method.
\end{abstract}

\section{Notation}

$A-$ channel cross section area, $\mathrm{m}^{2}$

$g-$ gravity, $\mathrm{kg} / \mathrm{s}^{2}$

$G$ - mass velocity, $G=\dot{\mathrm{m}} / \mathrm{A}, \mathrm{kg} /\left(\mathrm{s} \cdot \mathrm{m}^{2}\right)$

$K$ - physical property parameter

$L$ - length, $\mathrm{m}$

$\dot{m}$ - mass flow rate, $\mathrm{kg} / \mathrm{s}$

$p$ - pressure, $\mathrm{Pa}$

$R$ - friction factor or multiplier, $R=\Delta p_{T P f} / \Delta p_{l o}$

$v$ - specific volume, $\mathrm{m}^{3} / \mathrm{kg}$

$x$ - steam quality, $x=\dot{m}_{g} /\left(\dot{m}_{g}+\dot{m}_{l}\right)$

$X_{1}, X_{2}$ - Martinelli parameters

Greek symbols:

$\varphi$ - void fraction, $\varphi=A_{g} / A$

$\Phi$ - parameter proposed by Martinelli

$\mu$ - dynamic viscosity, $\mathrm{Pa} \cdot \mathrm{s}$

$\rho$ - density, $\mathrm{kg} / \mathrm{m}^{3}$

$\Theta$ - angle to horizontal plane, rad

subscripts:

$g$ - gas (steam)

go - gas only flow rate equal to that of two-phase flow

$l$ - liquid

lo - liquid only flow at a rate equal to that of two-phase flow

$T P f$ - two-phase friction term

\section{Introduction}

In a number of engineering systems it is important to be able to predict the pressure drop during boiling of twophase medium, such as water or other fluids. Generally, total two-phase pressure drop for flows in vertical channels consists of three components - frictional loss $\Delta p_{T P f}$, momentum change $\Delta p_{a}$ and elevation pressure drop $\Delta p_{h}$ arising from the effect of the gravitational force field. Thus

$$
\Delta p_{T P}=\Delta p_{T P f}+\Delta p_{a}+\Delta p_{h} .
$$

Generally, the current methods for modelling of twophase pressure drop fall into two categories: homogenous model and separated model. The latter is chosen in the paper for particular consideration. According to Collier and Thome [1], the total two-phase pressure drop may be written as

$$
\begin{aligned}
\Delta p_{T P}= & \frac{2 f_{l o} G^{2} v_{l} L}{D}\left[\frac{1}{x} \int_{0}^{x} R d x\right]+ \\
& G^{2} v_{l}\left[\frac{x^{2}}{\varphi}\left(\frac{v_{g}}{v_{l}}\right)+\frac{(1-x)^{2}}{(1-\varphi)^{2}}-1\right]+ \\
& \frac{L g \sin \Theta}{x} \int_{0}^{x}\left[\rho_{g} \varphi+\rho_{l}(1-\varphi)\right] d x
\end{aligned}
$$

It order to apply the above equation it is necessary to develop two expressions for multiplier $R$ and void fraction $\varphi$. Two different approaches are shown in the paper. The first one is the well-known LockhartMartinelli (LM) correlation for calculation of both quantities, the second one is developed by the present authors.

\footnotetext{
*Corresponding author: marian.trela@imp.gda.pl
} 


\section{The development of analytical formula for pressure multiplier $R$ and void fraction $\varphi$}

\subsection{The Lockhart-Martinelli Correlation}

This method was basically proposed by Lockhart and Martinelli in 1949 [2]. They introduced a few pertinent parameters. The first one is known as $\Phi_{l}^{2}$ parameter, defined as

$$
\Phi_{l}^{2}=\frac{(d p / d z)_{T P f}}{(d p / d z)_{l}}
$$

that is as a ratio of two-phase pressure gradient due to friction in a flow at mass rate equal to $\dot{m}=\dot{m}_{o}$ to the pressure gradient for a liquid stream flowing alone in the same channel at the mass flow rate $\dot{m}_{l}=(1-x) \dot{m}_{o}$.

The second parameter is known as Martinelli one and is defined as

$$
\mathrm{X}=\left[\frac{(d p / d z)_{l}}{(d p / d z)_{g}}\right]^{1 / 2}=\mathrm{X}_{1}
$$

what means that it is a ratio of pressure gradient due to friction of liquid phase flowing at mass rate equal to $\dot{m}=\dot{m}_{l}$ to the pressure gradient for a gas (vapour) stream flowing alone in the same channel at the mass flow rate $\dot{m}_{g}=x \dot{m}_{o}$. For each phase flowing separately in the channel at flow rate $\dot{m}_{l}$ or $\dot{m}_{g}$, the Martinelli parameter $X_{1}$ can be easily calculated as

$$
\mathrm{X}_{1}=\left(\frac{\rho_{g}}{\rho_{l}}\right)^{0.5}\left(\frac{\mu_{l}}{\mu_{g}}\right)^{0.1}\left(\frac{1-x}{x}\right)^{0.9} .
$$

It follows from (3) that

$$
\left(\frac{d p}{d z}\right)_{T P f}=\Phi_{l}^{2}\left(\frac{d p}{d z}\right)_{l} .
$$

The pressure gradient $(d p / d z)_{l}$ may by written as

$$
\left(\frac{d p}{d z}\right)_{l}=\left(\frac{d p}{d z}\right)_{l o}(1-x)^{2-n}
$$

where $n$ is the exponent in Blasius friction factor expression for pressure drop in single phase flow. Taking into account equations (6), (7) and definition of the multiplier $R$ one can obtain relation

$$
R=\frac{(d p / d z)_{T P f}}{(d p / d z)_{l o}}=(1-x)^{2-n} \Phi_{l}^{2} .
$$

Lockhart and Martinelli (LM) [2] have obtained experimental correlations for the parameter $\Phi_{l}$ and void fraction $\varphi$ in graphical forms as $\Phi_{l}=f\left(X_{1}\right)$ and $(1-\varphi)=f\left(X_{1}\right)$. The investigations were carried out for adiabatic horizontal two-phase flows of two-component systems (air-water, air-oil) at low pressure close to atmospheric one. Therefore, application of their graphs to other than the above mentioned conditions is not recommended. Another shortcoming of LockhartMartinelli correlations comes from their solely graphical form.

Two substantial improvements of LM correlations were done by Chisholm [3]. First, using theoretical approach, he proved that the usage of $\Phi_{l}$ and $X_{l}$ parameters is justified for the separate flow model of two-phase flow. Secondly, because the graphical form of LM correlation is cumbersome in practice, he proposed to replace the LM graphs by analytical correlation in the form

$$
\Phi_{l}^{2}=1+\frac{C}{\mathrm{X}_{1}}+\frac{1}{\mathrm{X}_{1}^{2}}
$$

where $C$ is a constant which depends on flow mechanisms that can exists during the simultaneous flow of a liquid and a gas (vapour) in the channel [3]. It is known that four types of flow mechanisms appear since the flow may be viscous (laminar) or turbulent. The particular values of $C$ are listed below for the given flow regime:

$\begin{array}{cc}\text { liquid } \quad \text { gas } & \mathrm{C} \\ \text { turbulent }- \text { turbulent }(\mathrm{tt}) & 20 \\ \text { viscous }- \text { turbulent }(\mathrm{vt}) & 12 \\ \text { turbulent }- \text { viscous }(\mathrm{vt}) & 10 \\ \text { viscous }- \text { viscous }(\mathrm{vv}) & 5\end{array}$

Using $n=0.25$ in (8) and $C=20$ in (9), for turbulentturbulent flow regime the multiplier $R$ takes the form

$$
R=(1-x)^{1.75}\left(1+\frac{20}{\mathrm{X}_{1}}+\frac{1}{\mathrm{X}_{1}^{2}}\right)
$$

which reproduces quite well the LM data for low pressure two-phase flow. Other, more recent modifications of LM correlation for micro- and macrochannels may be found in [4,5].

For high pressure and diabatic (with heat addition, e.g. at boiling) flows, the use of LM correlation (10) leads to large errors. Therefore the attempt was made by one of the present authors [6], to obtain analytical formulas for two-phase multiplier $R$ and void fraction $\varphi$. The present version of this approach differs significantly from that original one.

\subsection{Derivation of analytical formula for $R$ multiplier}

Experimental investigation of pressure drop during boiling of water in vertical tubes have been carried out by Martinelli and Nelson [7] using also Davidson et. al. [8] data. The data for multiplier $R$ and void fraction $\varphi$, Fig. 1 and Fig. 2, cover the whole two-phase domain from ambient pressure to critical one. The two-phase multiplier $R$ was presented versus a mass quality $x$ with pressure as a parameter, unfortunately in graphical form and in tables. Martinelli and Nelson [7] assumed that 
during flow boiling, the flow regime would always be "turbulent-turbulent".

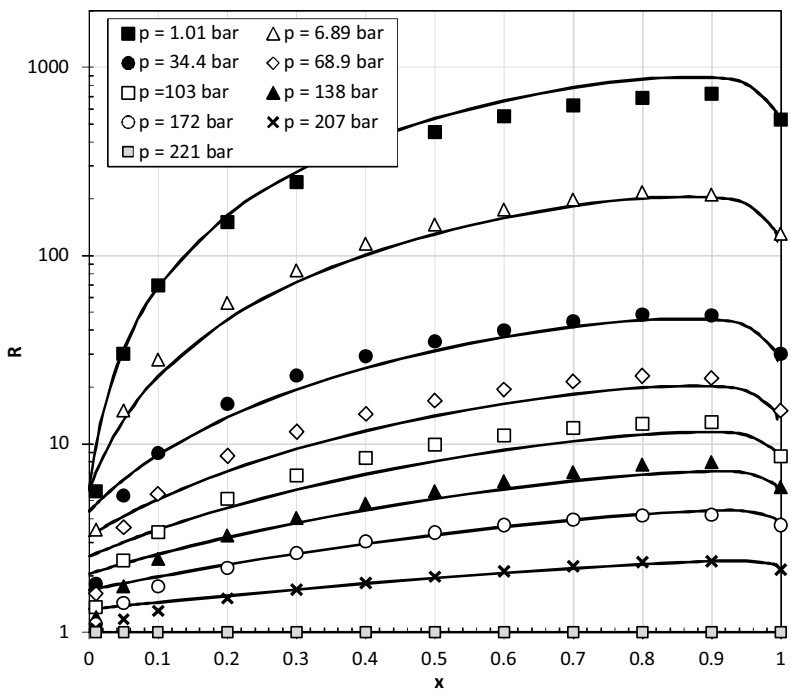

Fig. 1. Multiplier $R$ versus mass quality $x$ and pressure $p$ : symbols - experimental data [7], solid lines - correlation (20).

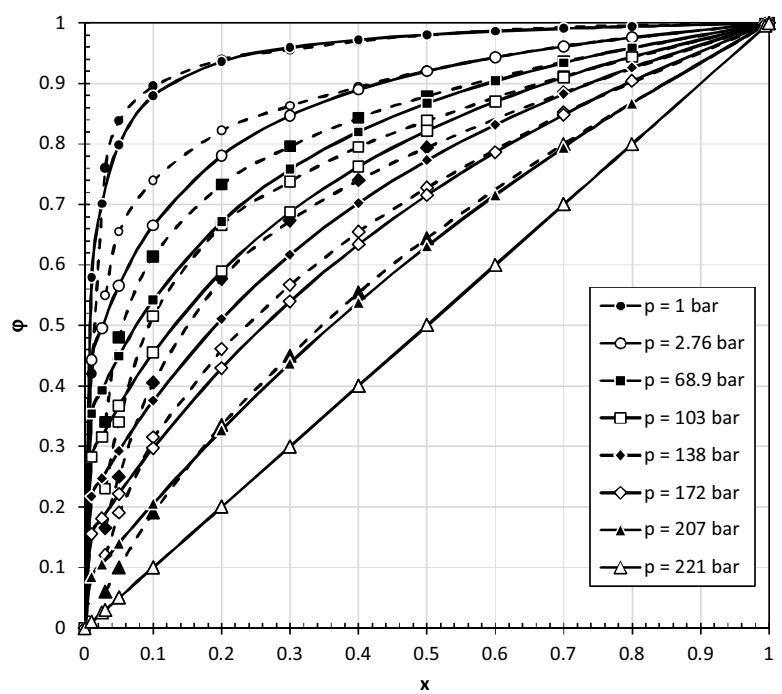

Fig. 2. Comparison of original (broken lines) and correlated (solid lines) values of void fraction $\varphi$ as a function of dryness fraction $x$ for selected values of pressure $p$. Original values are taken from Martinelli-Nelson experiments [7], approximated values are calculated according to (28).

The first aim of the present paper is therefore to develop mathematical formula for the multiplier $R$. The procedure starts from evaluation of the parameter $\Phi_{l}$ values using Fig. 1 for given pressure and steam quality $x$. Then from (8) it follows that

$$
\Phi_{l}=\sqrt{\frac{R}{(1-x)^{2-n}}} .
$$

For critical pressure $R=1$ so with $n=0.25$ the critical values of $\Phi_{l}$ equal to

$$
\Phi_{l c r}=\sqrt{\frac{1}{(1-x)^{1.75}}}
$$

Moreover, for the critical pressure the Martinelli parameter (5) reduces to

$$
X_{1 c r}=\left(\frac{1-x}{x}\right)^{0.9}
$$

since the physical properties of steam and liquid phases are the same. It may be shown that after extracting steam quality $x$ from (13) and substituting it into (12) one obtains critical value of parameter $\Phi_{l}$

$$
\Phi_{l c r}=\sqrt{\left(1+\frac{1}{\mathrm{X}_{1 c r}^{1.111}}\right)^{1.75}} \text {. }
$$

It was found earlier by Trela [6] that there is a relation between $\Phi_{l}$ and its critical value $\Phi_{l c r}$ at the same value of the Martinelli parameter, i.e. at condition that $X_{1}=i d e m$ for the critical pressure $p_{c r}$ and a pressure $p$. This relation was proposed in the form

$$
\Phi_{l}=D\left(\Phi_{l c r}\right)^{m}
$$

where $m$ is the slope of the $\log \left(\Phi_{l}\right)$ line with the reference to the abscissa and $D$ is a coefficient described by the relation

$$
D=\frac{\Phi_{l}}{\left(\Phi_{l c r}\right)^{m}} .
$$

It was found in [6] that formula (15) is valid within the range of steam quality $0.03 \leq x \leq 0.99$.

Using formula (14), the relation (15) can be rewritten in the following form

$$
\Phi_{l}=D\left(1+\frac{1}{X_{1}^{1.111}}\right)^{0.875 m}
$$

Relation (17) may be simplified if new Martinelli parameter $X_{2}$ is introduced, defined as

$$
X_{2}=X_{1}^{1.111}=\left(\frac{\rho_{g}}{\rho_{l}}\right)^{0.555}\left(\frac{\mu_{l}}{\mu_{g}}\right)^{0.111}\left(\frac{1-x}{x}\right) .
$$

Then

$$
\Phi_{l}=D\left(1+\frac{1}{X_{2}}\right)^{0.875 m}
$$

and accordingly to (8), for $n=0.25$, the multiplier $R$ takes the form

$$
R=(1-x)^{2-n} \Phi_{l}^{2}=(1-x)^{1.75} D^{2}\left(1+\frac{1}{X_{2}}\right)^{1.75 \cdot m}
$$


The above formula replaces the empirical diagram $R=f(x, p)$ given for boiling water by Martinelli Nelson [7]. Two parameters, $D$ and $m$, appear in the equation (20). In the former paper [6], the parameters $D$ and $m$ were expressed versus the property parameter $K$ in the graphical form. The parameter $K$ is defined by Eq. (21) which also means that $K$ is equal to the parameter $R$ when $x \rightarrow 1$,

$$
K=\frac{\left(\frac{d p}{d z}\right)_{g o}}{\left(\frac{d p}{d z}\right)_{l o}}=\left(\frac{\mu_{g}}{\mu_{l}}\right)^{0.25} \frac{\rho_{l}}{\rho_{g}} .
$$

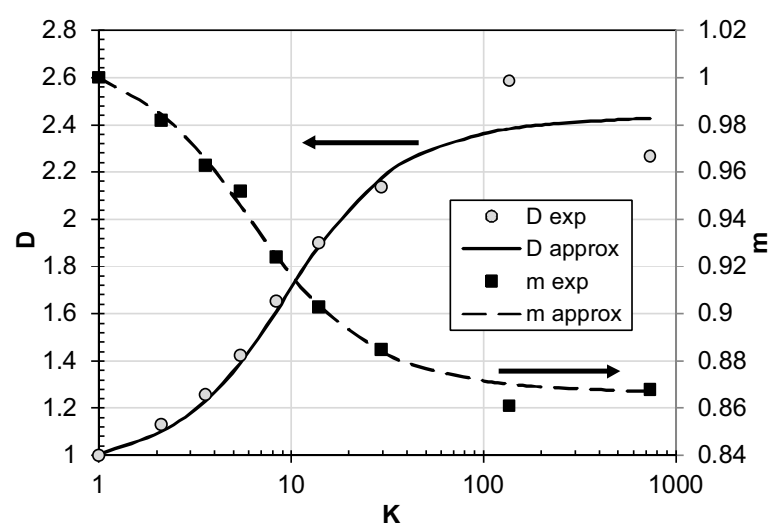

Fig. 3. Fitting of coefficients $D, m$ in multiplier $R$ correlation (20).

Figure 3 presents the variation of the parameters $D$ and $m$ versus the property parameter $K$. The former two parameters may be quite well approximated by the inverse trigonometric functions:

$$
D=1.18+0.8 \operatorname{arctg}\left(\frac{K-3}{9}\right)
$$

for $D$ parameter and

$$
m=1-0.085 \operatorname{arctg}\left(\frac{K-1}{6}\right)
$$

for the $m$ parameter.

\subsection{Derivation of analytical formula for void fraction $\varphi$}

Experimental investigations of the void fraction during flow boiling of water in vertical tubes have also been carried out by Martinelli and Nelson [7]. The data cover the whole two-phase domain from ambient pressure to critical one. The void fraction $\varphi$ was presented versus mass quality $x$ with pressure as a parameter in a graphical form, Figure 2. Procedure to develop an analytical formula for void fraction $\varphi$ is analogous to the previous one for the multiplier $R$. Under critical pressure, the physical properties of vapour and liquid phases are identical and the value of $\varphi$ becomes equivalent to quality $x$. Taking this into account one can write down that

$$
(1-\varphi)_{c r}=(1-x)_{c r}
$$

Martinelli parameter for the critical pressure is given by the formula (13). Extracting steam quality $x$ from (13) and substituting it into (24), one gets the expression

$$
(1-\varphi)_{c r}=\left(1+\frac{1}{X_{1}^{1.111}}\right)^{-1}=\left(1+\frac{1}{X_{2}}\right)^{-1}
$$

For the pressures lower than the critical, it was found in [6] that an analytical formula may be proposed for $\varphi$

$$
1-\varphi=E(1-\varphi)_{c r}^{k}
$$

where $k$ is the slope of straight lines in logarithmic coordinates and $E$ is a coefficient given by relation

$$
E=\frac{1-\varphi}{(1-\varphi)_{c r}^{k}} \text {. }
$$

Taking into account (25) and (26), it is easy to show that the void fraction formula takes the following form

$$
1-\varphi=E\left(1+\frac{1}{X_{2}}\right)^{-k}
$$

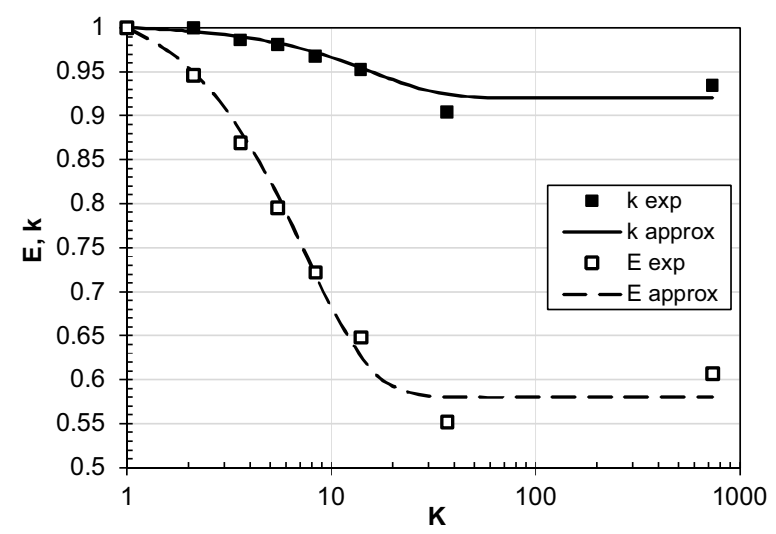

Fig. 4. Fitting of coefficients $k, E$ in void fraction approximation formula (28).

The values of the two coefficients $E$ and $k$ appearing in the above equation may be made dependent on the property parameter $K(21)$ and may be quite well approximated by the hyperbolic functions

$$
E=1-0.42 \tanh [0.11(K-1)]
$$

and

$$
k=1-0.08 \tanh [0.05(K-1)]
$$

as shown in Fig. 4. 


\subsection{Pressure drop similarity in two-phase flows}

Similarity criteria for the pressure drop in a flow can be based on the general theory of physical phenomena similarity [6]. It is required that geometrical similarity and the similarity of physical processes (described by relevant equations in dimensionless form together with boundary conditions) will be preserved.

Taking this into account, similarity criteria of two (geometrically similar) systems comprising two-phase flows include the equation describing the variation of the friction coefficient $R$ in the flow domain,

$$
R=(1-x)^{1.75} D^{2}\left(1+\frac{1}{X_{2}}\right)^{1.75 \cdot m}
$$

and the boundary conditions:

$$
\begin{array}{cc}
R=1 & \text { for } x=0, \\
R=K=\frac{(d p / d z)_{g o}}{(d p / d z)_{l o}}=\left(\frac{\mu_{g}}{\mu_{l}}\right)^{0.25} \frac{\rho_{l}}{\rho_{g}} & \text { for } x=1, \\
R=1 & \text { for } p=p_{c r} .
\end{array}
$$

If two two-phase fluid flows (I and II), one of which is water, are compared then it follows from the above relations that the two flows will be similar if the boundary conditions will be the same, as the Eq. (31) is valid for both flows, i.e. $R_{\mathrm{I}}=R_{\mathrm{II}}$. For the boundary conditions to be identical, the equality

$$
K_{I}=K_{I I}
$$

must be true for both flows considered. This means that the property parameter $K$ is a similarity number which must be the same for the two systems. The conclusion from these considerations is such that by making the coefficients $D$ and $m$ in Eq. (31) functions of the physical parameter $K$, that equation can be applied to calculate the frictional pressure drop in two-phase flows of various working fluids, not only for water.

\section{Validation of the proposed method}

The validation of the above method may be done in two ways. Firstly, as illustrated in Figure 1, the experimental values of multiplier $R$ (symbols) can be compared with their approximated values (solid lines) calculated according to (20). It is seen that analytical approximation fits quite well the Martinelli-Nelson data. Similarly, Figure 2 shows the comparison of Martinelli-Nelson data with analytical approximation of void fraction $\varphi$ (28). In this case, there is also good agreement between original Martinelli-Nelson data and the analytical approximation. It has to be noted that the approximation applied is valid for a steam quality in the range $0.03 \leq x \leq 0.99$.

Second validation of the method comes from the comparison of the experimental data of the multiplier $R$ for Freon 21 with theoretical values calculated from Eq. (20) with coefficients $D$ and $m$ given by (22) and (23), Fig. 5 and Fig. 6. The experimental investigation was carried out by Trela [9] in Laboratory of IF-FM in
Gdańsk. The influence of mass velocity $G$ on the multiplier $R$ was also investigated.

Figure 5 shows also the prediction of the $R$ multiplier according to well-known formulas of Lottes [10] and Levy [11]:

- Lottes formula $R=\left(\frac{1-x}{1-\varphi}\right)^{2}$,

- Levy's formula $R=\frac{(1-x)^{1.75}}{(1-\varphi)^{2}}$.

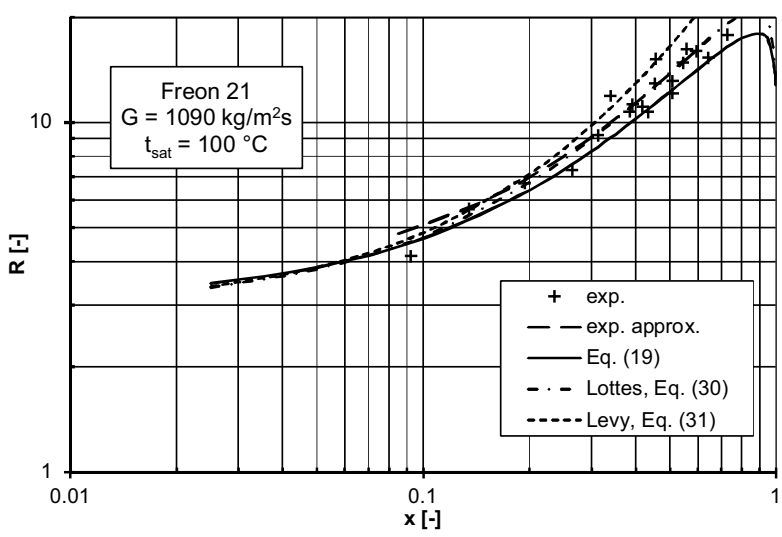

Fig. 5. Calculated (solid line) and experimental values (cross marks) of multiplier $R$ versus quality $x$ for Freon 21 at saturation temperature $t_{\text {sat }}=100{ }^{\circ} \mathrm{C}$ and mean mass velocity $G=1090 \mathrm{~kg} / \mathrm{m}^{2} \mathrm{~s}$.

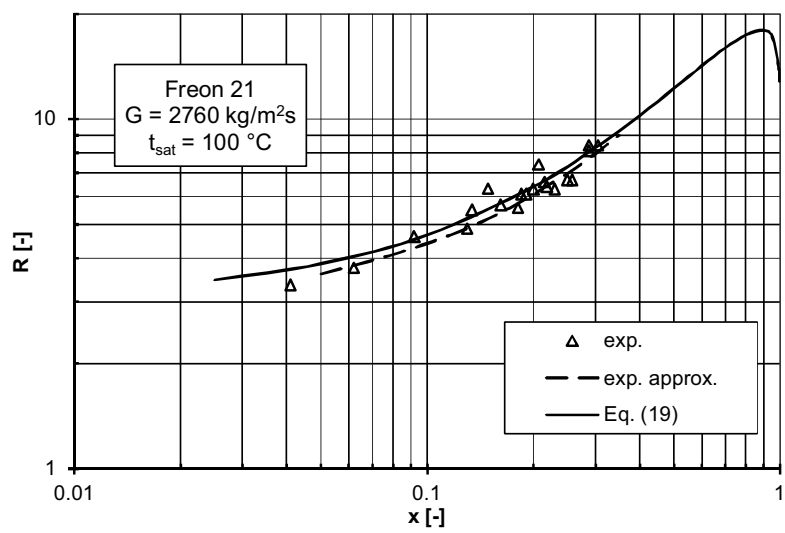

Fig. 6. Calculated (solid line) and experimental values (triangles) of multiplier $R$ versus quality $x$ for Freon 21 at saturation temperature $t_{\text {sat }}=100{ }^{\circ} \mathrm{C}$ and mean mass velocity $G=2760 \mathrm{~kg} / \mathrm{m}^{2} \mathrm{~s}$.

Figure 6 presents experimental results of $R$ multiplier for higher mass velocity $G=2760 \mathrm{~kg} / \mathrm{m}^{2} \mathrm{~s}$. The dashed lines in Figures 5 and 6 show average values of $R$ evaluated from the experiments. It is seen that $R$ is decreasing when the mass velocity increases. This tendency is confirmed by literature, e.g. [1]. The experimental data of multiplier $R$ obtained for Freon 21 [9] suggest that effect of mass velocity on $R$ may be written as 


$$
R_{\text {mod }}=R \Omega
$$

where the correction factor $\Omega$ is expressed as

$$
\Omega=\left(\frac{1400}{G}\right)^{0.25}
$$

in the range $G=1000 \div 2500 \mathrm{~kg} / \mathrm{m}^{2} \mathrm{~s}$. A systematic investigation of mass velocity influence on pressure drop in two-phase flow was conducted by Baroczy [12] for a variety of fluids. His results show very complex dependence of the correction factor $\Omega$ not only on the mass velocity $G$ but also on property index and steam quality. The Baroczy correction factor $\Omega_{B}$ was presented in the form of graphs as a function of property index $K_{B}$ defined as

$$
K_{B}=\left(\frac{\mu_{l}}{\mu_{g}}\right)^{0.2} \frac{\rho_{g}}{\rho_{l}} .
$$
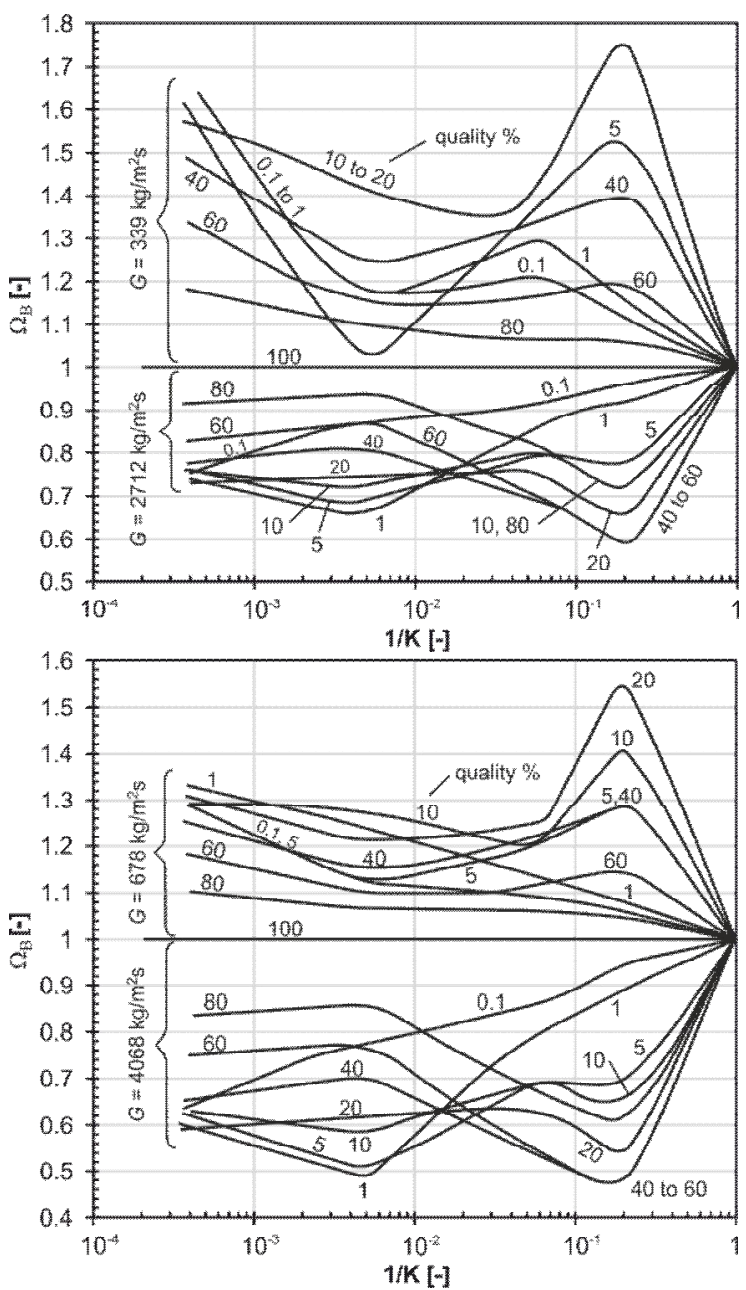

Fig. 7. Mass velocity correction factor $\Omega_{B}$ as a function of property parameter $1 / K$ and steam quality $x$ for selected values of mass velocity $G$, adapted from Baroczy [12]

It is seen that Baroczy property index (37) is similar to the property parameter $K(20)$, namely

$$
K_{B} \approx 1 / K \text {. }
$$

Therefore it is proposed to adapt Baroczy correction factor $\Omega_{B}$ to use with the multiplier $R$ in (35) as a more general formula than (36). Due to complex representation of $\Omega_{B}$, it is impossible to find a simple approximation function allowing direct numerical calculations so its values should be read from the plots shown in Fig. 7.

Table 1. Initial conditions for pressure drop measurements in R134a two-phase flow [13].

\begin{tabular}{|c|c|c|c|}
\hline Case No. & $G\left[\mathrm{~kg} / \mathrm{m}^{2} \mathrm{~s}\right]$ & $p[\mathrm{bar}]$ & $p / p_{\text {crit }}$ \\
\hline 1 & 1200 & 10 & 0.25 \\
\hline 2 & 1200 & 25 & 0.62 \\
\hline 3 & 1200 & 36 & 0.89 \\
\hline 4 & 1800 & 10 & 0.25 \\
\hline 5 & 1800 & 25 & 0.62 \\
\hline 6 & 1800 & 36 & 0.89 \\
\hline
\end{tabular}
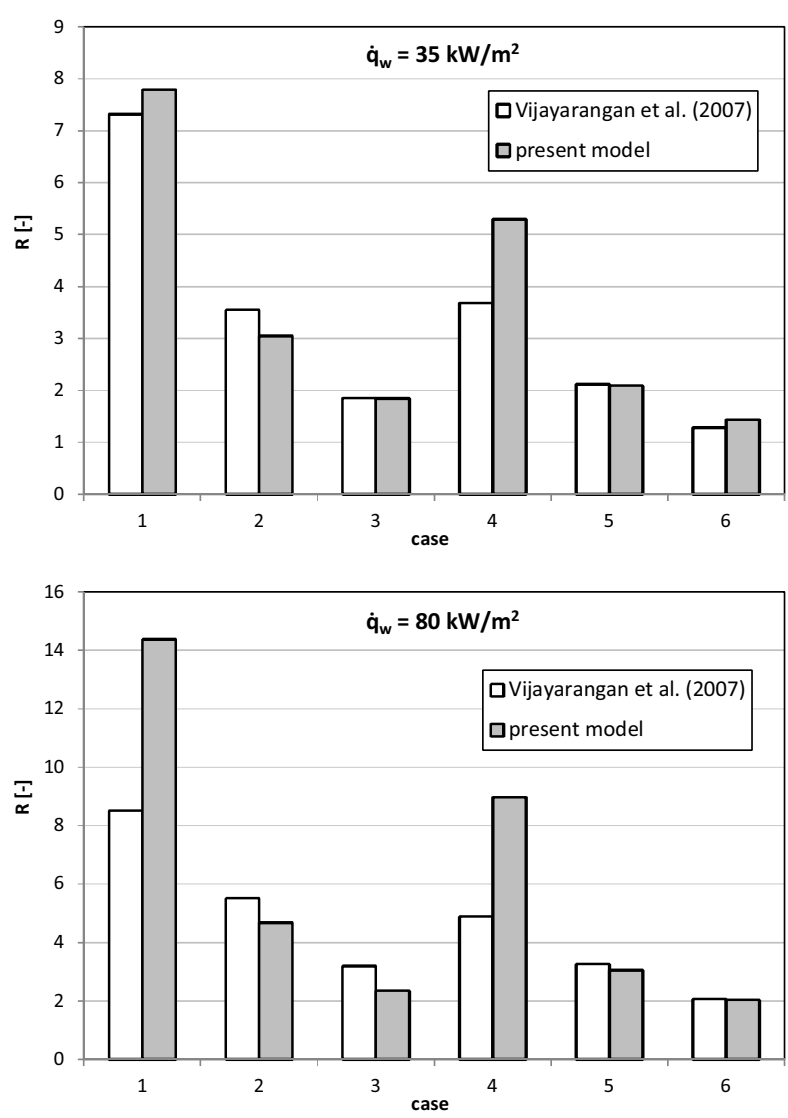

Fig. 8. Comparison of multiplier $R$ calculated with present model, i.e. Eq. (20) with Baroczy correction factor $\Omega_{B}$, and derived from Vijayarangan et al. [13] measurements for R134a.

The predictions of the proposed method of multiplier $R$ calculation (20) with the correction factor $\Omega_{B}$ were compared with experimental data for R134a refrigerant reported by Vijayarangan et al. [13]. For this purpose, a total of 12 Vijayarangan et al. measurements of frictional pressure drop in a vertical tube of $12.7 \mathrm{~mm}$ internal diameter were selected. Initial flow conditions for the comparison, which are a combination of two 
mass velocity values and three pressure values, are named as Case 1 to 6 and defined in the Table 1 . In each case, two heat flux $\dot{q_{w}}$ values $\left(35\right.$ or $\left.80 \mathrm{~kW} / \mathrm{m}^{2}\right)$ were supplied to the R134a flow from the uniformly heated tube wall.

The comparison results are shown in Fig. 8. Good agreement is achieved between the Vijayarangan et al. data and the present model predictions, except for the cases with $p=10$ bar and $\dot{q}_{w}=80 \mathrm{~kW} / \mathrm{m}^{2}$, where the model clearly overestimates the $R$ value.

\section{Conclusions}

The paper presents a simple methodology to transform Martinelli-Nelson graphical forms of two-phase friction multiplier $R$ and void fraction $\varphi$ into their equivalent analytical expressions.

Comparison of Martinelli-Nelson data of $R$ and $\varphi$ with their analytical approximations, Eq. (20) and (28), shows very good agreement, Fig. 1 and Fig 2.

Non-dimensional property parameter $K$, Eq. (21), was introduced to allow the use of the above method to calculate multiplier $R$ and void fraction $\varphi$ for other fluids than water, at the condition that $K$ for water has to be equal to $K$ for that other fluid.

Experimental investigation of pressure drop of Freon 21 in vertical tube $\left(d_{i n}=11.6 \mathrm{~mm}\right)$ during boiling proved the validity of Eqs. (20) and (28), as well as the usefulness of the property parameter $K$.

The above investigation also showed the effect of mass velocity on the multiplier $R$. Formula (35) was proposed to take this effect into account for Freon 21. For other fluids Baroczy [12] correction factor was adapted in the form of graphs shown in Fig. 7 and checked against the experimental data for R134a [13].

This research has been supported by National Science Centre within the Project No. 2012/05/B/ST8/02742

\section{References}

[1] J.G. Collier, J.R. Thome, Convective Boling and Condensation, Clarendon Press, Oxford (1994)

[2] P.A. Lockhart, R.C. Martinelli, Proposed correlation of data for isothermal two-phase two- component flow in pipes, Chemical Engineering Progress, 45, No. 1, 39-48 (1949)

[3] D. Chisholm, A theoretical basis for the LockhartMartinelli correlation for two-phase flow, Int. J. Heat Mass Transfer, 10, 1767-1778 (1967)

[4] Y.S. Muzychka, M.M. Awad, Asymptotic generalizations of the Lockhart-Martinelli method for two phase flows, Transactions of the ASME Journal of Fluids Engineering, 132, Issue 3, 031302-1 - 031302-12 (2010)

[5] S.K. Saha, G.P. Celata, Heat transfer and pressure drop in flow boiling in microchannels, Springer, Cham, Switzerland (2016)

[6] M. Trela, A new approach to predicting pressure drop in two-phase flows, Trans. of the Institute of Fluid Flow Machinery (IMP PAN), 64, 41-57 (1974)

[7] R.C. Martinelli, D.B. Nelson, Prediction of pressure drop during forced circulation boiling of water, Trans. ASME, 70, 695-702 (1948)

[8] W.F. Davidson, P.H. Hardie, C.R. Humphrey, Studies of heat transmission through boiler tubing at pressures from 500 to 3300 pounds, Trans. ASME, 65, 553-591 (1943)

[9] M. Trela, Experimental investigations of the pressure drop of freon 21 during flow boiling in vertical pipes, Trans. of the Institute of Fluid Flow Machinery (IMP PAN), 64, 59-78 (1974)

[10] P.A. Lottes, M. Petrick, F. Marchattere, Lecture notes on heat extraction from boiling water power reactors, USA EC Report ANL-6063 (1959)

[11] S. Levy, Steam slip theoretical prediction from momentum model, J. Heat Transfer, Trans. ASME series C, 82 (1960)

[12] C.J. Baroczy, A systematic correlation for twophase pressure drop, Chemical Engineering Progress Symposium Series, 62, No. 64, 232-249 (1966)

[13] B.R. Vijayarangan, S. Jayanti, A.R. Balakrishnan, Pressure drop studies on two-phase flow in a uniformly heated vertical tube at pressures up to the critical point, Int. J. Heat Mass Transfer, 50, 1879-1891 (2007) 